\title{
Visual marking across eye blinks
}

\author{
David E. Irwin • Glyn W. Humphreys
}

Published online: 17 October 2012

(C) Psychonomic Society, Inc. 2012

\begin{abstract}
Visual search for a conjunction target can be made efficient by presenting one initial set of distractors as a preview, prior to the onset of the other items in the search display Watson \& Humphreys (Psychological Review 104:90-122, 1997). However, this "preview advantage" is lost if the initial items are offset for a brief period before onsetting again with the search display Kunar, Humphreys, \& Smith (Psychological Science 14:181-185, 2003). Researchers have long disputed whether the preview advantage reflects a process of internally coding and suppressing the old items or of the onset of the new items capturing attention Donk \& Theeuwes (Perception \& Psychophysics 63:891-900, 2001). In this study, we assessed whether an internally driven blink (in which participants close their eyes) acts in the same manner as an external blink produced by offsetting and then onsetting the preview. In the novel blink conditions, participants searched feature, conjunction, and preview displays after being cued to blink their eyes. The search displays were presented during the eye blink, and so were immediately available once participants opened their eyes. Having participants make an eye blink generally slowed search but had no effect on the search slopes. In contrast, imposing an externally driven blink disrupted preview search. The data indicated that visual attention can compensate for internally driven blinks, and this does not lead to the loss of the representations of distractors across time. Moreover, efficient preview search occurred when the search items had no abrupt onsets, demonstrating that onsets of new search items are not critical for the preview benefit.
\end{abstract}

D. E. Irwin $(\bowtie)$

Department of Psychology, University of Illinois,

603 E. Daniel Street,

Champaign, IL 61820, USA

e-mail: irwin@illinois.edu

G. W. Humphreys

Department of Experimental Psychology, University of Oxford,

Oxford OX2 3UD, UK
Keywords Attention $\cdot$ Memory $\cdot$ Visual search

In a complex visual world, participants can use dynamic temporal context to help segment relevant from irrelevant stimuli occurring at different time intervals. This has been modeled using preview search (Watson \& Humphreys, 1997). In preview search, participants are presented with an initial set of distractors that remain in the field when a new set of search items appears to define a final conjunction display. When the initial preview occurs $400 \mathrm{~ms}$ or so prior to the new search items, search is as efficient as when only the new search items appear. Watson and Humphreys originally proposed that participants took advantage of the temporal delay between the preview and search items to inhibit the preview so that previewed items no longer competed for selection with the new stimuli. They termed this process "visual marking." An alternative view is that the attention of participants is captured by the onsets of the new search items (Donk \& Theeuwes, 2001), removing any impact of the old, previewed stimuli on search.

The ability of participants to use the temporal interval between the old and new items to facilitate search is disrupted if a blank interval (an externally driven "blink") is introduced after the preview, with the preview reappearing along with the new search display (Kunar, Humphreys, \& Smith, 2003). This may occur because the removal of the preview eliminates its internal representation, so that there is no longer a representation to suppress. Alternatively, the reonsetting of the old and new items means that the new stimuli no longer have an onset advantage (Donk \& Theeuwes, 2001), or it may be that the common onsets of both sets of items lead to temporal grouping of the stimuli, overriding any marking of the old items (Humphreys, Riddoch, Nys, \& Heinke, 2002). In addition, inserting an "attentional blink" (having participants identify a target and then presenting the search display) also selectively disrupts preview search (Olivers \& Humphreys, 2002), suggesting that continued attention to the old items is required in 
order to capitalize on the temporal dynamics of preview conditions (see also Humphreys, Watson, \& Jolicœur, 2002).

Here, we compared the effects of real (internally driven) eye blinks with the effects of externally driven blinks produced by offsetting and then onsetting the stimuli. Several possible results might have occurred. One possibility was that a real blink could "wipe clean" any representation of the preview, in which case we would expect the preview advantage in search to be disrupted, perhaps akin to the effects with an externally driven blink. A second possibility was that there would be no loss of the representation of the preview, and consequently, no common onset of the old and new items, since stimulus-driven offsets would be absent in this case. This might allow the preview advantage to emerge. This last result would be of theoretical interest, because it would indicate that the preview advantage is not contingent on new search items being defined by common onsets, going against the "onset capture" account of preview search.

\section{Method}

\section{Participants}

A group of 20 students from the University of Illinois community (7 male and 13 female) participated. Their ages ranged from 19 to 26 years $(M=20.4)$. The participants reported normal or corrected-to-normal vision and were naive as to the purpose of the experiment. They received payment for participating in a single, 90 -min session.

\section{Stimuli}

In the conjunction search conditions, participants searched for a blue $H$ (RGB value: $0,51,255$ ) among green $H$ s (RGB value: $0,102,51)$ and blue $A$ s (RGB value: $0,51,255)$. The feature search conditions involved a search for a blue $H$ among blue $A$ s. On each trial, participants had to decide whether the bar of the blue $H$ was higher or lower than the midpoint of the vertical sides of the letter. The green $H$ distractors also had bars in positions above or below their vertical midpoints, thereby making the distractor $H$ s similar to the target $H$ and forcing conjunction search (Kunar et al., 2003). Each letter display contained four, eight, or 16 items on conjunction search trials, and two, four, or eight items on feature search trials. The items were randomly positioned within a virtual $7 \times 7$ grid that subtended $9.3^{\circ} \times 11.4^{\circ}$. Each letter subtended $0.8^{\circ} \times 1.4^{\circ}$.

\section{Apparatus}

The stimuli were presented on a 21 -in. monitor with resolution of $800 \times 600$ pixels and a refresh rate of $85 \mathrm{~Hz}$. Eye movements and blinks were recorded with an EyeLink II video-based eyetracker (SR Research Ltd., Mississauga, Ontario, Canada) with a temporal resolution of $500 \mathrm{~Hz}$, a spatial resolution of $0.1^{\circ}$, and a pupil-size resolution of $0.1 \%$ of pupil diameter. The output of the eyetracker was analyzed online to detect eye blinks. Each data sample from the eyetracker contained a timestamp in milliseconds, the velocity and the position of the eye, and the area of the pupil. An eye blink was defined as a period of missing pupil for at least six consecutive milliseconds. Blink onset and blink offset were defined to correspond to the beginning and ending of the period of missing pupil. Custom $\mathrm{C}$ code was written to display stimuli and collect responses. Participants' heads were stabilized with a chinrest, fixed at $57 \mathrm{~cm}$ from the computer monitor, and the display background was light gray (luminance $=86.3 \mathrm{~cd} / \mathrm{m}^{2}$ ). The participants made manual responses by pressing buttons on a Microsoft Sidewinder digital game controller interfaced with the eyetracking computer.

Design and procedure

Each participant was tested in seven conditions. Three of the conditions (see Fig. 1) involved eye blinks, and thus were of major interest to this investigation. In these conditions, participants began each trial by pressing a button on the game controller while fixating on a drift-correcting dot. A blank white screen was presented for $500 \mathrm{~ms}$, and then one of three things happened, depending on the experimental condition. Of prime interest was the blink-contingent preview

\section{Blink-Contingent Preview}

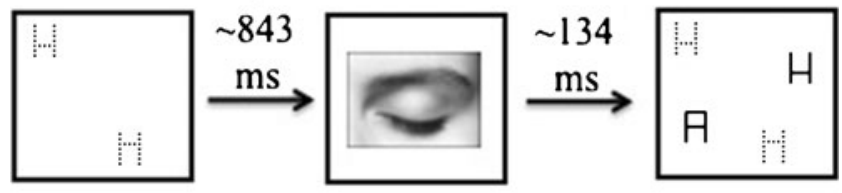

Blink-Contingent Conjunction

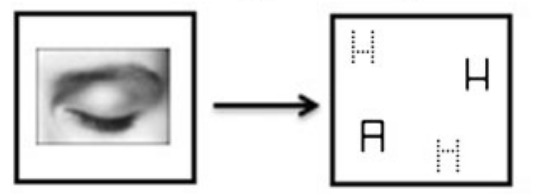

\section{Blink-Contingent Feature}

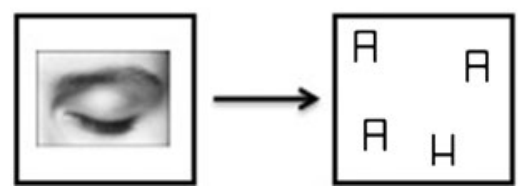

Fig. 1 Example displays for the blink-contingent conditions. Letters shown here with dotted lines were green in the displays that the participants viewed, while letters shown with solid lines were blue 
condition, in which a display of green $H$ s was presented. After $500 \mathrm{~ms}$, a tone was presented for $100 \mathrm{~ms}$, which cued participants to make an eye blink. When the blink was detected, the blue $H$ and blue $A$ s were added to the green $H$ s while the eyes were closed, so that the green $H \mathrm{~s}$, blue $H$, and blue $A$ s were visible on the screen when the eyes reopened. The average blink latency (defined with respect to tone onset) was $343 \mathrm{~ms}$ ( $S E=13.0 \mathrm{~ms}$ ), which means that on average, participants had an 843 -ms preview of the green $H$ s before the eye blink. The average blink duration (defined as the time period when the pupil was completely covered by the eyelid) was $134 \mathrm{~ms}$, so the display (and everything else) was not visible during this time. Because all of the search items were visible on the screen when the eyes reopened, no abrupt stimulus-driven onsets were associated with the presentation of the target display. Of interest was whether visual marking would survive the eye blink - that is, whether a preview effect would be found under these conditions.

To assess this, two additional blink conditions were conducted. In the blink-contingent conjunction condition, following drift correction and the 500-ms white screen, a tone was presented for $100 \mathrm{~ms}$. Participants blinked as soon as they heard the tone, and when the blink was detected, the blue $H$, blue $A$ s, and green $H \mathrm{~s}$ were presented simultaneously while the eyes were closed, and thus were visible on the screen when the eyes reopened. This condition provided a measure of search efficiency when no preview was presented, so if visual marking does not survive an eye blink, performance in the blink-contingent preview condition should resemble that in the blink-contingent conjunction condition. In contrast, if visual marking fully survives an eye blink, search slopes in the blink-contingent preview condition should be half those in the blink-contingent conjunction condition, because half of the items in the display (i.e., the preview items) would not compete for selection. The participants also completed a blinkcontingent feature condition, whose procedure was identical to that of the blink-contingent conjunction condition, except that during the blink, only the blue $H$ and blue $A$ s were presented simultaneously while the eyes were closed. In all conditions, the participant had to indicate whether the bar of the blue $H$ was higher or lower than the midpoint of the vertical sides of the letter, and response times (RTs, measured from blink offset - the moment when the pupil became uncovered) and accuracy were recorded.

Participants completed four additional conditions that did not involve eye blinks (see Fig. 2); these served as partial replications of other studies in the visual-marking literature (e.g., Watson \& Humphreys, 1997) and also provided comparative information for performance in the blink conditions. In the no-blink conditions, after drift correction, a blank white screen was presented for $500 \mathrm{~ms}$, followed by a 100-ms warning tone. Then one of four things happened, depending on the experimental condition. In the feature
Feature

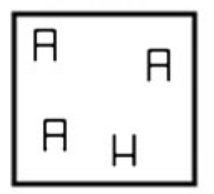

\section{Conjunction}
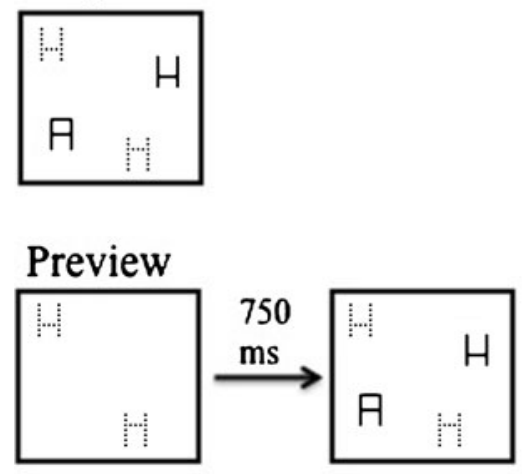

\section{Preview with delay}

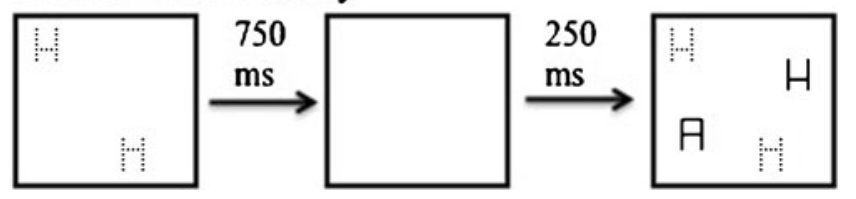

Fig. 2 Example displays for the no-blink conditions. Letters shown here with dotted lines were green in the displays that the participants viewed, and letters shown with solid lines were blue

condition, the blue $H$ and blue $A$ s were presented simultaneously. In the conjunction condition, the blue $H$, blue $A$ s, and green $H \mathrm{~s}$ were presented simultaneously. In the preview condition, green $H \mathrm{~s}$ appeared for $750 \mathrm{~ms}$, and then the blue $H$ and blue $A$ s were added to the green $H$ s. In the previewwith-delay condition, green $H \mathrm{~s}$ appeared for $750 \mathrm{~ms}$, the display went blank for $250 \mathrm{~ms}$, and then the green $\mathrm{Hs}$ reappeared along with the blue $H$ and blue $A$ s. The participants indicated whether the bar of the blue $H$ was higher or lower than the midpoint of the vertical sides of the letter, and RTs and accuracy were recorded.

The comparison of the blink-contingent preview condition with the no-blink preview and no-blink preview-with-delay conditions was of interest. If the period of time when the eyes are closed during an eye blink acts like clearing the screen and inserting a delay before presenting the target display, then performance in the blink-contingent preview condition should resemble that in the no-blink preview-plus-delay condition. In contrast, if eye blinks are ignored or taken into account by the perceptual system, then visual marking should persist across the eye blink, and performance in the blink-contingent preview condition should resemble that in the no-blink preview condition. 
Each of the seven conditions was presented in a separate block, with 80 trials in each block. The first 20 trials in each block were discarded as practice, and the remaining 60 trials contained 20 trials at each display size (two, four, or eight in the feature conditions, and four, eight, or 16 in the conjunction conditions). The order of the blocks was randomized across participants.

\section{Results}

One participant failed to complete the experiment in the time allotted, and another had very high error rates (more than $30 \%$ ) in some conditions, so the data from these two participants were dropped from the analysis.

Error rates (averaged over the remaining 18 participants) as a function of condition and display size are shown in Table 1. Display size in the feature conditions was matched to that in the conjunction conditions in order to facilitate comparison with the preview conditions (Watson \& Humphreys, 1997). No significant effects were found in a two-way withinparticipants analysis of variance on these data with Display Size, $F(2,34)=0.5, M S E=.001, p>.5$, and Condition, $F(6,102)=1.9, M S E=.001, p>.05$, as the main factors. The interaction between display size and condition was also not significant, $F(12,204)=1.1, M S E=.001, p>.3$.

Figure 3 shows the mean correct RTs as a function of display size for each condition. The data are shown separately for the no-blink and blink-contingent trials for ease of inspection. The RTs for correct trials only were entered into a two-way within-participants analysis of variance with Display Size and Condition as the main factors. The main effect of display size was significant, $F(2,34)=212.7, M S E=$ $9,412, p<.001$; as expected, RTs increased as display size did. The main effect of condition was also significant, $F(6,102)=$ $10.8, M S E=37,661, p<.001$. In general, RTs were slower in the blink conditions than in the corresponding no-blink conditions, most likely because blinks suppress vision (e.g.,

Table 1 Mean percentage error rates as a function of condition and display size

\begin{tabular}{llll}
\hline \multirow{2}{*}{ Condition } & \multicolumn{3}{l}{ Display Size } \\
\cline { 2 - 4 } & 4 & 8 & 16 \\
\hline No-blink feature & 0.6 & 0.3 & 1.1 \\
No-blink conjunction & 1.7 & 0.0 & 0.8 \\
No-blink preview & 3.1 & 1.1 & 1.4 \\
No-blink preview with delay & 2.2 & 1.7 & 2.8 \\
Blink-contingent feature & 1.4 & 1.4 & 1.1 \\
Blink-contingent conjunction & 0.8 & 2.2 & 0.8 \\
Blink-contingent preview & 1.7 & 1.7 & 1.9 \\
\hline
\end{tabular}
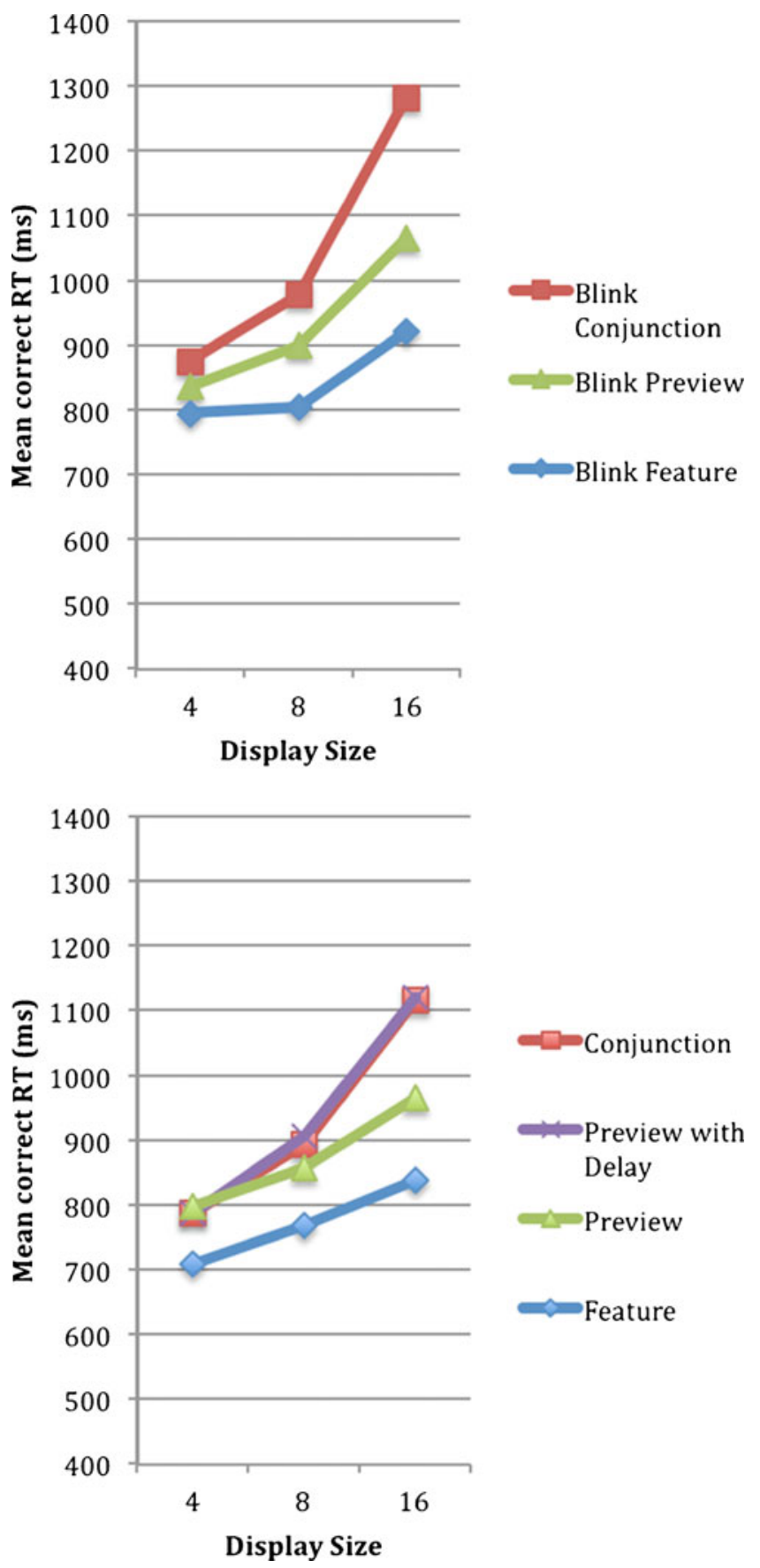

Fig. 3 Mean correct response times (RTs, in milliseconds) as a function of display size for the blink-contingent conditions (top) and the noblink conditions (bottom)

Volkmann, Riggs, \& Moore, 1980) and requiring participants to blink in response to a tone imposes some cognitive demand. The most straightforward way to determine the effect of these factors was to compare RTs in the blink-contingent feature and conjunction conditions with RTs in the no-blink feature and conjunction conditions, because the only difference between these pairs of conditions was that a blink initiated the stimulus onset in the blink-contingent conditions. RTs were slower in 
the blink-contingent feature condition $(839 \mathrm{~ms}$ ) than in the noblink feature condition (772 ms), and RTs were slower in the blink-contingent conjunction condition $(1,045 \mathrm{~ms})$ than in the no-blink conjunction condition (933 ms); the $95 \%$ confidence interval for the condition main effect was $\pm 55.3 \mathrm{~ms}$, so both differences were significant. Taking the average of the differences, it appears that blinking slowed overall RTs by approximately $90 \mathrm{~ms}$.

Of major interest, of course, was the interaction between condition and display size. This interaction was significant, $F(12,204)=14.7, M S E=4,111, p<.001$. To examine the interaction, the slope of the RT by display size function was calculated for each condition and each participant, and these slopes were then analyzed in an ANOVA with Condition as the only factor. The effect of condition was once again significant, $F(6,102)=19.9, M S E=78.7, p<.001$. Paired-samples $t$ tests were then conducted to examine the comparisons of interest. To determine whether blinking affected the dynamics of the search process, slopes in the no-blink feature and conjunction conditions were compared with those in the blink-contingent feature and conjunction conditions. The slope for blink-contingent feature trials $(11.1 \mathrm{~ms})$ was not significantly different from the slope for no-blink feature trials (10.6 ms), $t(17)=0.2, S D=11.4, p$ > .80. Similarly, the slope for blink-contingent conjunction trials $(34.5 \mathrm{~ms})$ was not significantly different from the slope for no-blink conjunction trials $(27.4 \mathrm{~ms}), t(17)=1.7, S D=$ $17.4, p>.10$. Thus, although blinking slowed RTs overall, it appeared to have little effect on the search process per se.

Let us now turn to the main question of interest, whether visual marking survives an eye blink. The slope in the blinkcontingent preview condition (19.3 ms) was significantly less than the slope in the blink-contingent conjunction condition (34.5 ms), $t(17)=3.8, S D=16.9, p<.001$. The $95 \%$ confidence interval around the slope for the blink-contingent preview condition extended from 14.8 to $23.6 \mathrm{~ms}$; this included $17.3 \mathrm{~ms}$, or half of the slope of the blink-contingent conjunction condition. This result indicates that the previewed items did not compete for selection when the target display was presented, demonstrating that visual marking does survive an eye blink. This conclusion was supported by comparing the slope of the blink-contingent preview condition with the slopes of the no-blink preview and preview-with-delay conditions. Although preview durations were somewhat longer in the blink-contingent preview condition $(843 \mathrm{~ms})$ than in the no-blink preview conditions $(750 \mathrm{~ms})$, Watson and Humphreys (1997) showed that the preview effect changes very little for preview durations longer than $400 \mathrm{~ms}$, so this was unlikely to hinder the comparison. The slope in the blinkcontingent preview condition (19.3 ms) was significantly less than the slope of the no-blink preview-with-delay condition (27.6 ms), $t(17)=2.9, S D=12.1, p=.01$, but it did not differ significantly from the slope of the no-blink preview condition
(14.0 ms), $t(17)=2.0, S D=11.0, p>.06$. This result indicates that eye blinks do not affect search in the same way that clearing the display and inserting a delay does; rather, it appears that eye blinks are ignored or taken into account by the perceptual system, so that visual marking persists across the eye blink. Because the target display was presented while the eyes were closed, no abrupt stimulus-driven onsets were associated with the target onset. This supports the conclusion that top-down inhibition of previewed items, rather than the occurrence of abrupt onsets for new items, is responsible for the phenomenon of visual marking.

One potential cause for concern is that the mean blink duration $(134 \mathrm{~ms})$ was shorter than the blank period used in the no-blink preview-with-delay condition $(250 \mathrm{~ms})$. Perhaps visual marking survived the eye blink only because the blank period between the preview and target displays was relatively short. To investigate this possibility, we conducted two additional analyses. In one, we correlated the mean blink duration for each participant in the blink-contingent preview condition with the participant's slope in that condition. Longer blink durations correspond to longer delays between the preview and target displays, so slopes should increase as blink duration increases if visual marking persists only across short blink durations. The mean blink durations across individual participants ranged from 41 to $286 \mathrm{~ms}$, while the slopes ranged from 4.1 to $38.6 \mathrm{~ms}$; the correlation between mean blink duration and slope was only .007 , however, indicating that the persistence of visual marking across eye blinks did not depend on blink duration. In the second analysis, we analyzed only the data from the six participants who had the longest blink durations (we used six participants for reasons of statistical power, and because the mean of these six was approximately equal to the blank period used in the no-blink preview-withdelay condition). The mean blink duration across these six participants was $221 \mathrm{~ms}$, with a range from 156 to $286 \mathrm{~ms}$. Analysis of the slopes for these six participants showed that the effect of condition was significant, $F(6,30)=10.3, M S E=$ $43.8, p<.001$. Paired-sample $t$ tests replicated the analysis of the full data set in every respect. The slope for blinkcontingent feature trials $(11.2 \mathrm{~ms})$ was not significantly different from the slope for no-blink feature trials $(8.8 \mathrm{~ms}), t(5)=$ $0.7, S D=8.2, p>.5$. Likewise, the slope for blink-contingent conjunction trials $(32.6 \mathrm{~ms})$ was not significantly different from the slope for no-blink conjunction trials $(27.5 \mathrm{~ms}), t(5)=$ $0.8, S D=15.8, p>4$. The slope in the blink-contingent preview condition $(17.9 \mathrm{~ms})$ was significantly less than the slope in the blink-contingent conjunction condition (32.6 ms), $t(5)=3.6, S D=10.0, p<.02$. The $95 \%$ confidence interval around the slope for the blink-contingent preview condition extended from 14.2 to $21.6 \mathrm{~ms}$, which includes $16.3 \mathrm{~ms}$, or half of the slope of the blink-contingent conjunction condition. The slope in the blink-contingent preview condition $(17.9 \mathrm{~ms})$ was significantly less than the slope in the no-blink preview-with- 
delay condition $(24.2 \mathrm{~ms}), t(5)=3.4, S D=4.6, p=.02$, but it did not differ significantly from the slope in the no-blink preview condition $(17.2 \mathrm{~ms}), t(5)=0.3, S D=5.3, p>.7$. In sum, this analysis showed that visual marking survives an eye blink even when the blink duration is approximately equal to the delay that we used in the no-blink preview-with-delay condition.

\section{Discussion}

Our data were clear. While inserting an externally driven blink after the preview disrupted preview search (replicating the results of Kunar et al., 2003), there was no effect of a real (internally driven) blink on search efficiency: Performance was as efficient in the blink condition as in the standard preview condition, and for both conditions, performance was more efficient than in the conjunction baseline. The results showed that an internal blink does not have the same effect as an external blink in terms of its effect on dynamic visual search. There may be several reasons for this.

One possibility is that an internally driven blink may have a minimal correlation with event changes in the world, whereas an externally driven blink can signal a change in the state of the environment. This external change may reset the system so that a representation of the prior display is not maintained. In contrast, the lack of correlation between an internally driven blink and an environmental change may mean that a prior representation of the world is maintained, to help guide behavior in the unchanged environment. Here, marking of the preview would allow search to be guided more efficiently to a search target by reducing attentional competition from the old, inhibited distractors. A problem for this account is that Kunar et al. (2003) showed that, even when the preview is removed, traces of it can be found if it reappears slightly ahead of the search items, thus preventing the items from being grouped by common onset. Kunar, Humphreys, Smith, and Watson (2003) also found a preview advantage when the old items moved (and offset) behind an occluder and then reappeared at the same time as the new items. In these cases, offset of the preview did not abolish the preview advantage.

A more likely reason for the preview advantage under realblink conditions is that, under conditions of the real blink, the items in the search display do not onset together-they are already in the field when the participant's eyes open. When there is no common onset of the old and new items, the tendency to group them should be reduced, and under these conditions, marking of the old items could still dominate. Given other results of the preview advantage surviving a temporal gap when common onsets are prevented (Kunar et al., 2003), we believe that this common-onset account provides a plausible explanation for our results.
Research in other domains has also found that blinks do not have the same effects on perception as does blanking the stimulus. For example, Volkmann et al. (1980) found that visual perception is suppressed before, during, and after blinks, so that the blackout that occurs while the pupil is covered is hardly noticed, whereas external interruptions of visual input, such as turning out the lights in a room, are quite noticeable, even if they are briefer than an eye blink. Deubel, Bridgeman, and Schneider (2004) and Higgins, Irwin, Wang, and Thomas (2009) found that eye blinks do not have the same effect as stimulus blanking in judgments of visual direction. Deubel et al. hypothesized that the oculomotor signal that accompanies an eye blink allows the perceptual system to distinguish between internal and external sources of temporary object disappearance, so that display blanking and eye blinking have different effects on perception.

One further implication of our results relates to the status of different accounts of preview search. As we have noted, one account is that the preview advantage occurs because attention is captured by the new onsets (Donk \& Theeuwes, 2001). However, in our study the new items did not onset at a different time from the old items, but rather, all reappeared together after a real blink had occurred. This finding indicates that it is not necessary to define the new search items by novel onsets in order for a preview advantage to occur. This same conclusion has been reached in studies in which the search items are presented under isoluminant conditions, where luminance onsets are not present to define the new items (Braithwaite, Hulleman, Watson \& Humphreys, 2006; Braithwaite, Humphreys, Watson \& Hulleman, 2005). Though inconsistent with an onset-capture account, the data are compatible with the idea that there is inhibitory marking of old distractors, which are known to be irrelevant to the subsequent search task. The results suggest that old items do not have to remain present in the display to remain marked, and so marking survives a real, internally driven blink.

\section{References}

Braithwaite, J. J., Hulleman, J., Watson, D. G., \& Humphreys, G. W. (2006). Is it impossible to inhibit isoluminant items, or does it simply take longer? Evidence from preview search. Perception \& Psychophysics, 68, 290-300. doi:10.3758/BF03193676

Braithwaite, J. J., Humphreys, G. W., Watson, D. G., \& Hulleman, J. (2005). Revisiting preview search at isoluminance: New onsets are not necessary for the preview advantage. Perception \& Psychophysics, 67, 1214-1228. doi:10.3758/BF03193554

Deubel, H., Bridgeman, B., \& Schneider, W. X. (2004). Different effects of eyelid blinks and target blanking on saccadic suppression of displacement. Perception \& Psychophysics, 66, 772-778. doi:10.3758/BF03194971

Donk, M., \& Theeuwes, J. (2001). Visual marking beside the mark: Prioritizing selection by abrupt onsets. Perception \& Psychophysics, 63, 891-900. doi:10.3758/BF03194445 
Higgins, J. S., Irwin, D. E., Wang, R. F., \& Thomas, L. E. (2009). Visual direction constancy across eyeblinks. Attention, Perception, \& Psychophysics, 71, 1607-1617. doi:10.3758/APP.71.7.1607

Humphreys, G. W., Riddoch, M. J., Nys, G., \& Heinke, D. (2002a). Unconscious transient binding by time: Neuropsychological evidence from anti-extinction. Cognitive Neuropsychology, 19, 361380 .

Humphreys, G. W., Watson, D. G., \& Jolicœur, P. (2002b). Fractionating the preview benefit in search: Dual-task decomposition of visual marking state by timing and modality. Journal of Experimental Psychology. Human Perception and Performance, 28, 640-660. doi:10.1037/0096-1523.28.3.640

Kunar, M. A., Humphreys, G. W., \& Smith, K. J. (2003a). History matters: The preview benefit in search is not onset capture. Psychological Science, 14, 181-185. doi:10.1111/1467-9280.t011-01439
Kunar, M. A., Humphreys, G. W., Smith, K. J., \& Watson, D. G. (2003b). When reappearance is old news: Visual marking survives occlusion. Journal of Experimental Psychology. Human Perception and Performance, 29, 185-198. doi:10.1037/00961523.29.1.185

Olivers, C. N. L., \& Humphreys, G. W. (2002). When visual marking meets the attentional blink: More evidence for top-down, limitedcapacity inhibition. Journal of Experimental Psychology. Human Perception and Performance, 28, 22-42. doi:10.1037/00961523.28.1.22

Volkmann, F. C., Riggs, L. A., \& Moore, R. K. (1980). Eyeblinks and visual suppression. Science, 207, 900-902.

Watson, D. G., \& Humphreys, G. W. (1997). Visual marking: Prioritizing selection for new objects by top-down attentional inhibition of old objects. Psychological Review, 104, 90-122. doi:10.1037/0033-295X.104.1.90 